\title{
Prevalence of Coma-Recovery Scale-Revised signs of consciousness in patients in minimally conscious state.
}

\author{
Wannez Sarah ${ }^{1}$, Gosseries Olivia ${ }^{1}$, Azzolini Deborah ${ }^{1}$, Martial Charlotte $^{1}$, \\ Cassol Helena ${ }^{1}$, Aubinet Charlène ${ }^{1}$, Annen Jitka ${ }^{1}$, Martens Géraldine ${ }^{1}$, \\ Bodart Olivier ${ }^{1}$, Heine Lizette ${ }^{1}$, Charland-Verville Vanessa ${ }^{1}$, Thibaut \\ Aurore $^{1}$, Chatelle Camille ${ }^{1,2}$, Vanhaudenhuyse Audrey ${ }^{1,3}$, Demertzi \\ Athena $^{1}$, Schnakers Caroline ${ }^{4,5}$, Donneau Anne-Françoise ${ }^{6}$, Laureys \\ Steven $^{1}$
}

1) Coma Science Group, GIGA-Consciousness and Neurology Department, University of Liège, Liège, Belgium

2) Department of Neurology, Massachusetts General Hospital, Harvard Medical School, Boston, MA

3) Department of Algology, University Hospital of Liège, Liège, Belgium.

4) Department of Neurosurgery, University of California, Los Angeles, CA, USA.

5) Research Department, Casa Colina Hospital and Centers for Healthcare, Pomona, CA, USA

6) Biostatistic Unit, Department of Public Health, University of Liège, Liège, Belgium

Word count: 3523

Corresponding author

Sarah Wannez

coma@chu.ulg.ac.be

Coma Science Group, GIGA-Consciousness

$\mathrm{CHU}-\mathrm{B} 34$

Quartier Hopital

Avenue de l'Hopital, 11

4000 Liège

Belgium 


\section{Prevalence of Coma-Recovery Scale-Revised signs of consciousness in patients in minimally conscious state.}

Different behavioural signs of consciousness can distinguish patients with an unresponsive wakefulness syndrome from patients in minimally conscious state (MCS). The Coma Recovery Scale-Revised (CRS-R) is the most sensitive scale to distinguish the different altered states of consciousness and eleven items detect the MCS. The aim of the present study is to document the prevalence of these items. We analysed behavioural assessments of 282 patients diagnosed in MCS based on the CRS-R. Results showed that some items are particularly frequent among patients in MCS, namely fixation, visual pursuit, and reproducible movement to command, which were observed in more than $50 \%$ of patients. These responses were also the most probably observed items when the patients only showed one sign of consciousness. On the other hand, some items were rarely or never observed alone, e.g., object localization (reaching), object manipulation, intelligible verbalization, and object recognition. The results also showed that limiting the CRS-R assessment to the 5 most frequently observed items (i.e., fixation, visual pursuit, reproducible movement to command, automatic motor response and localisation to noxious stimulation) detected $99 \%$ of the patients in MCS. If clinicians have only limited time to assess patients with disorders of consciousness, we suggest to evaluate at least these five items of the CRS-R.

Keywords: Coma Recovery Scale-Revised, minimally conscious state, consciousness, behavioural assessment, severe brain injury. 


\section{Introduction}

After a period of coma, patients who survive but do not fully recover awareness are diagnosed in disorders of consciousness (DOC). When they open their eyes without showing any behavioural sign of consciousness, they are diagnosed in an unresponsive wakefulness syndrome, previously coined as "vegetative state" (UWS/VS; Laureys et al., 2010). Some of them will evolve into a minimally conscious state (MCS; Giacino et al., 2002), characterized by the presence of subtle but reproducible signs of consciousness. Those patients can be subcategorized into two distinct entities (Bruno, Vanhaudenhuyse, Thibaut, Moonen, \& Laureys, 2011): on one hand, MCS minus patients showing low-level purposeful behaviours (e.g., visual pursuit, object localization (reaching), automatic motor response), and on the other hand, MCS plus patients showing a movement to command. Those behavioural differences were confirmed by a group analysis of their cerebral metabolism, with a preservation of the language areas in the MCS plus group and not in the MCS minus group (Bruno et al., 2012). Finally, patients who are able to functionally communicate and/or to functionally use different objects have emerged from MCS (EMCS; Giacino et al., 2002). Misdiagnosis between all these altered states of consciousness has important prognosis, therapeutic and ethical implications. First, the prognosis is affected by the diagnosis. For example, patients who are MCS one month after the brain injury have greater chance to recover within the first year, than patients who are UWS/VS at this point (Bruno, et al., 2011). Second, patients with residual consciousness may experience pain and emotions, while this is not the case for UWS/VS patients (Boly et al., 2005). The diagnosis might therefore influence analgesic treatment and end-of-life decisions, as shown among a large sample of paramedical and medical professionals (Demertzi et al., 2011; Demertzi et al., 2009). 
The first and main tool to diagnose patients with DOC is behavioural assessment. This is very challenging and reported rates of misdiagnosis are around $40 \%$ (Andrews, Murphy, Munday, \& Littlewood, 1996; Schnakers et al., 2009; Stender et al., 2014). The use of a standardized scale can reduce the misdiagnosis when compared to clinical diagnosis provided by the medical staff (Schnakers et al., 2009). A number of behavioural scales are designed to assess patients with DOC, including the Glasgow Coma Scale (Teasdale \& Jennett, 1974), the Sensory Modality Assessment and Rehabilitation Technique (Gill-Thwaites, 1997), the Wessex Head Injury Matrix (Shiel et al., 2000), and the Full Outline of UnResponsiveness (Wijdicks, Bamlet, Maramattom, Manno, \& McClelland, 2005). However, among all the available scales, the American Congress of Rehabilitation Medicine (Seel et al., 2010) preferably recommends the use of the Coma Recovery Scale-Revised, because of its good psychometric properties (CRS-R; Giacino, Kalmar, \& Whyte, 2004). The CRS-R includes 6 subscales, assessing different functions with various numbers of hierarchically arranged items. These subscales comprise auditory, visual, motor, oromotor/verbal functions, communication and arousal. The total score is composed of the sum of the maximum score obtained in each subscale, and can help distinguishing UWS/VS from MCS patients (Bodien, Carlowicz, Chatelle, \& Giacino, 2016), although it cannot completely supply to a qualitative analysis of the subscores. Indeed, a patient can be diagnosed in MCS only if specific items, indicating the MCS, are reached by the patient. Apart from the arousal, each subscale contains items that assess MCS.

A previous study highlighted the importance of testing visual and motor subscales when diagnosing patients with DOC, because $27 \%$ of patients were detected in MCS only by the visual subscale (i.e., no other subscale could detect a reproducible and voluntary response), and $8 \%$ only by the motor subscale (Estraneo et al., 2014). 
Fifty-two percent of patients showing an evolution to MCS in rehabilitation centres were detected by only one subscale ( $9 \%$ by motor subscale, $43 \%$ by visual subscale). Moreover, $86 \%$ of patients showed at least one visual conscious behaviour, confirming the importance of the visual subscale (Bagnato et al., 2016). In another study, 55\% of patients only show visual conscious behaviours, while $20 \%$ of patients showed signs of consciousness in both visual and motor subscale (Noé et al., 2012).

According to the guidelines, each CRS-R subscale should be administered in a specific order, with the higher-level items tested first. If patients are correctly responding to these items, the lower-level items should not be administered. Indeed, it is assumed that patients would automatically be able to succeed these lower-level items if they succeeded higher-level items. However, as suggested by some authors, the presence of high-level behaviours does not guarantee that the patient is able to complete lower-level behaviours, due to different neurological structures that underlie these behaviours (Sattin et al., 2015).

The aims of this study is to document the frequency of each CRS-R item that denotes MCS and to identify the most frequently observed items in order to guide clinicians in their behavioural assessments.

\section{Methods}

We retrospectively extracted the clinical profile of severely brain-injured patients diagnosed in MCS by our tertiary centre at the University Hospital of Liège in the last 10 years. For each patient, we included the first CRS-R in which all signs of MCS have been tested. For example, if a visual pursuit was observed, the fixation was nonetheless tested. Moreover, if one combination of subscores was considered as impossible according to the CRS-R guidelines (Chatelle et al., 2016; Giacino et al., 2004), the 
assessment was not considered as complete, and thus excluded. All CRS-R assessments were conducted by skilled and experienced neuropsychologists, when patients were free of sedative drugs (i.e., without anaesthetic agents that would make them unconscious). This study was approved by the ethics committee of the University of Liège, and written informed consent was obtained from each patient's legal surrogate.

Eleven CRS-R items are considered as signs of a MCS: consistent movement to command, reproducible movement to command, object recognition, object localization (reaching), visual pursuit, fixation, automatic motor response, object manipulation, localization to noxious stimulation, intelligible verbalization, and intentional communication. We scored these items as present or absent for each patient, as instructed in the CRS-R guidelines (for instance, "visual pursuit" is present if the patient follows a mirror for 45 degrees without loss of fixation on at least 2 occasions in any of the 4 directions). Note that behavioural responses have to be reproducible to be scored as present. Comparisons between the aetiology groups (i.e., traumatic, anoxic, nontraumatic non-anoxic) were performed with Chi-square tests. Mann-Whitney U tests were used to compare the time since onset according to the presence or absence of the different items. The percentages of patients detected by a subset of CRS-R items were evaluated for the 3,4 and 5 most frequent items. Results were considered significant at $\mathrm{p}<0.05$, corrected for multiple comparisons.

\section{Results}

Two hundred eighty-two patients in MCS were included in the study (mean age $=47 \pm 19$ years old; mean time since onset $=1.7 \pm 3.8$ years; 120 traumatic, 74 anoxic, and 88 nontraumatic non-anoxic; 96 women; 145 MCS plus). In the whole cohort, the three most frequently observed items were fixation (57\%), visual pursuit (52\%) and reproducible 
movement to command (51\%). The percentages related to each item are reported in figure 1. No difference was observed between the aetiology groups (all $\mathrm{p}>0.05$ ). Patients with visual pursuit had a longer time since onset than patients without visual pursuit $(\mathrm{Z}=2.95, \mathrm{p}=0.034)$. Patients with localization to noxious stimulation had shorter time since onset than patients without this behaviour $(\mathrm{Z}=4.43, \mathrm{p}<0.001)$.

Among the 282 patients, 115 (41\%) only showed one sign of consciousness. The same 3 items were the most frequently observed in this case, but in different proportions: reproducible movement to command (35\%), fixation $(27 \%)$, and visual pursuit (20\%). The percentages related to each item are reported in figure 2.

\section{INSERT FIGURES 1 AND 2 AROUND HERE}

One hundred and five patients (37\%) showed some higher-level behaviours while they did not show lower-level behaviours within the same subscale.. For example, a visual pursuit was detected, but no fixation.

At the subscale level, a sign of consciousness was observed in the auditory subscale in 144 patients $(51 \%)$, in the visual subscale in 207 patients $(73 \%)$, in the motor subscale in 99 patients $(35 \%)$, in the oromotor subscale in 11 patients $(4 \%)$, and in the communication subscale in 30 patients $(11 \%)$. In 152 patients, only one subscale detected a MCS, which was the visual subscale in 89 patients (59\%), the auditory in 41 patients $(27 \%)$, the motor in 21 patients $(14 \%)$ and the oromotor in 1 patient $(1 \%)$.

Finally, based on those results, we determined the percentages of patients that would have been correctly detected as MCS if only a subset of CRS-R items had been computed. Different possible subsets were assessed: the 3 most frequent items 
(movement to command, fixation and visual pursuit); the 4 most frequent items (adding localization to noxious stimulation); and the 5 most frequent items (adding automatic motor response). If only these 5 CRS-R items had been tested, clinicians would have been able to correctly identify $99 \%$ of patients in MCS. The percentages and confidence intervals for the 3 different subsets are reported in table 1 .

\section{INSERT TABLE 1 AROUND HERE}

\section{Discussion}

The aim of the study was to document the frequency of the observed CRS-R items reflecting consciousness in patients in MCS, when all those items were tested in the same session for a patient. We here wanted to focus on the signs of consciousness that can distinguish patients in UWS/VS and MCS, knowing that the misdiagnosis rate between these patients can reach $41 \%$ (Schnakers et al., 2009). We showed that the most frequently observed items were fixation, visual pursuit, and reproducible movement to command. We also observed that $41 \%$ of patients only showed one sign of consciousness during the whole assessment. In this case, the same three items were detected as most frequent, although in a different order. The aetiology did not have any impact on the presence of the different signs of consciousness. Visual pursuit has however been previously documented as more frequent among patients with traumatic brain injury or vascular accidents than in patients with anoxic or hypoxic aetiology (Dolce et al., 2011), a difference that we did not reproduce here.

A relationship was observed between time since onset and some CRS-R items. Patients with visual pursuit tended to have longer time since onset, which is in line with a previous study that showed reappearance of visual pursuit with time (Dolce et al., 
2011). Patients with localization to noxious stimulation showed shorter time since onset, suggesting that this behaviour might disappear as time goes on. One possible explanation for this motor degradation could be spasticity, because it is known to positively correlate with time (Thibaut et al., 2015). The patients' level of consciousness, especially in a chronic stage, might then be underestimated and one can erroneously consider the patient as unresponsive and unconscious, with the negative implications that we know, for example on medical decisions (Demertzi et al., 2009). We thus emphasize the importance of treating limb spasticity in patients with disorders of consciousness, for instance with physical therapy, medication and splints (Thibaut et al., 2013; Thibaut et al., 2015). The level of consciousness might also be underestimated if the patient suffers from visual impairment. Even if visual behaviours have been reported frequently in this study and in previous ones (Bagnato et al., 2016; Estraneo et al., 2014; Noé et al., 2012), clinicians can not only rely on these items to identify patients in MCS.

In accordance with previous studies, we also found a slight percentage of patients who only showed voluntary motor responses, but the new finding here is the prevalence of conscious auditory responses, thus it is important to test movements to command in all patients. The auditory subscale did not appear alone in the previous studies comparing behavioural responses of patients in MCS (Bagnato et al., 2016; Estraneo et al., 2014; Noé et al., 2012). Aphasia might be incriminated when a patient does not show any movement to command (Majerus, Bruno, Schnakers, Giacino, \& Laureys, 2009). When stroke patients suffering from aphasia but without any disorders of consciousness were assessed with the CRS-R, their level of consciousness was underestimated in $25 \%$ of the cases (Schnakers et al., 2015). However, surprisingly, movement to command was present in all aphasic patients (if not systematic, at least 
reproducible), while they failed in more complex items such as functional communication or use of objects. This result implies that even when the patients suffer from global aphasia, they are still able to follow simple commands. Besides aphasia and visual impairment, other troubles should be considered in the absence of movement to command, such as motor impairment or attentional deficits. To circumvent the motor impairment, new techniques using brain-computer interface are of great help for clinicians. For example, motor imagery with electroencephalography recording proved to be efficient in detecting a response to command in the electrical activity of some patients that did not clinically show movement to command (e.g., Cruse et al., 2011). A last source of underestimation is the lack of consistency. Indeed, CRS-R guidelines requires a minimum of three responses out of four to score a response to command, for example. Patients who only succeed two out of four trials are thus considered as nonresponsive. This is inherent to the CRS-R scoring system.

Our results also showed that if a higher-level behaviour was observed in a patient, lower-level items were not systematically present, in line with recent literature (Sattin et al., 2015). This implies that patients with automatic motor response, for example, will not necessarily show object manipulation. If one wants to fully describe the clinical profile of a patient, it is important to assess all the items, and not to consider that the lower-level items are automatically present if the high-level items are scored.

Three items were observed alone in rare cases, namely object localization (reaching), object manipulation, and intelligible verbalization (less than $1 \%$ of the cases when only one sign of consciousness was observed). This suggests that most of the time, patients who are able to show these signs of consciousness can also correctly respond to other CRS-R items that denote MCS. On the other hand, some items are never observed alone. This is inherent to the CRS-R guidelines, as intentional 
communication and object recognition can never appear without a reproducible movement to command. This is in fact one of the impossible combinations of CRS-R scoring (Chatelle et al., 2016). Systematic movement to command also automatically implies that the patient has reproducible movement to command.

Apart from the retrospective aspect of the present study, some limitations should be mentioned. We only included patients' behavioural assessments in a specific moment, without repeated measures or follow-up. Future studies should examine whether the clinical profile is changing over time (short and long-term), and whether some items appear or disappear after a while. Longitudinal studies should also provide more evidence that the time since onset influences the clinical profile in the same patient. Moreover, assessment of spasticity could also be included in the behavioural measures to test the hypothesis of a progressive motor impairment due to this affection. Another factor to take into account is that different experimenters assessed the patients, which could be considered as a source of bias. However, the CRS-R is recognized for its good properties regarding inter-rater reliability (Giacino et al., 2004; Seel et al., 2010). One study highlighted that this reliability is modulated by the experience of the examiner (Lovstad et al., 2010). In our case, all examiners who assessed the patients were trained and experienced. Another limitation, which is inherent to the clinical practice, is that the order of the items is not randomized. Language impairments and aphasia could also be seen as a problem (Majerus et al., 2009). Even when patients are known to be fully conscious, language deficits can introduce a bias, and underestimate the level of consciousness (Schnakers et al., 2015). However, this limitation is inherent to the CRS-R and not specific to our study.

Despite these limitations, the present results were obtained in a large and varied sample of patients in MCS, and hence should be considered reliable. To our knowledge, 
this is the first time that prevalence of each and every CRS-R item denoting a MCS is documented. We suggest that when time is lacking or when the patient is particularly tired, the CRS-R assessment could be limited to 3, 4 or 5 items, depending on the risk the clinician agrees on. If the clinician assesses movement to command, visual pursuit, fixation, localization to noxious stimulation, and automatic motor response, almost $99 \%$ of patients will be correctly diagnosed as MCS, according to the present sample. If no sign of consciousness is detected with the most frequently observed items, the clinician should then perform the complete CRS-R. Reducing the length of the behavioural assessment might be in general more efficient, as patients can easily be tired after a long testing. Clinicians could also do a complete CRS-R assessment of patients with DOC, but begin with those frequently observed items, to increase the probability to observe a sign of residual consciousness. Needless to say that communication and functional use of objects would also have to be tested, as they detect patients having emerged from the MCS.

Future studies should look for any link between the items that denote MCS and the other low-level items of the CRS-R. A recent study has documented the different possible combinations (Chatelle et al., 2016), but on the basis of the maximum score in each subscale. Such a work with all the items tested as present or absent might improve our understanding on the different clinical profiles of patients. Future studies should also investigate neuroimaging results such as brain metabolism, electrical activity or hemodynamic responses that are associated with each CRS-R item in order to help understanding the neural correlates of subtle signs of consciousness in patients in MCS.

\section{Conclusion}


The CRS-R is currently the most sensitive scale to distinguish patients with DOC, and 11 items are designed to detect patients in MCS. Some of them are more likely to be observed (i.e., fixation, visual pursuit, reproducible movement to command, localization to noxious stimulation and automatic motor response), while some are infrequently or never observed alone (i.e., object manipulation, object localization (reaching), intelligible verbalization, systematic movement to command, intentional communication, and object recognition), due to infrequent prevalence or due to the CRS-R guidelines. Based on these results, we propose to clinicians who want to quickly assess a patient, because of various clinical reasons, to limit their assessments to a subset of CRS-R items. Indeed, testing visual pursuit, fixation, movement to command, localization to noxious stimulation and automatic motor response might already detect 99\% of patients in MCS.

\section{Acknowledgements}

The authors thank the patients and their families, the University and University Hospital of Liège, the French Speaking Community Concerted Research Action (ARC - 06/11 340), the Belgian National Funds for Scientific Research (FRS-FNRS), the WallonieBruxelles International, the James McDonnell Foundation, Mind Science Foundation, IAP research network P7/06 of the Belgian Government (Belgian Science Policy), the European Commission, the Public Utility Foundation 'Université Européenne du Travail', "Fondazione Europea di Ricerca Biomedica", the Bial Foundation, Human Brain Project (EU-H2020-fetflagship-hbp-sga1-ga720270), and Luminus project (EUH2020-fetopen-ga686764). LH, OB, CM, CA are research fellows, OG, VCV, AT, AD are post-doctoral fellow and SL is research director at FRS-FNRS. 
References

Andrews, K., Murphy, L., Munday, R., \& Littlewood, C. (1996). Misdiagnosis of the vegetative state: retrospective study in a rehabilitation unit. $B M J, 313,13-16$. http://doi.org/10.1136/bmj.313.7048.13

Bagnato, S., Boccagni, C., Sant'Angelo, A., Fingelkurts, A. A. A., Fingelkurts, A. A. A., \& Galardi, G. (2016). Longitudinal assessment of clinical signs of recovery in patients with unresponsive wakefulness syndrome after traumatic or nontraumatic brain injury. Journal of Neurotrauma, 33, 1-5.

Bodien, Y. G., Carlowicz, C. A., Chatelle, C., \& Giacino, J. T. (2016). Sensitivity and specificity of the coma recovery scale-revised total score in detection of conscious awareness. Archives of Physical Medicine and Rehabilitation, 97(3), 490-492. http://doi.org/10.1016/j.apmr.2015.08.422

Boly, M., Faymonville, M.-E., Peigneux, P., Lambermont, B., Damas, F., Luxen, A., ... Laureys, S. (2005). Cerebral processing of auditory and noxious stimuli in severely brain injured patients: Differences between VS and MCS. Neuropsychological Rehabilitation, 15(3-4), 283-289.

Bruno, M.-A., Ledoux, D., Vanhaudenhuyse, A., Gosseries, O., Thibaut, A., \& Laureys, S. (2011). Pronostic des patients récupérant du coma. In C. Schnakers \& S. Laureys (Eds.), Coma et états de conscience altérée (pp. 17-30). Paris: Springer.

Bruno, M.-A., Majerus, S., Boly, M., Vanhaudenhuyse, A., Schnakers, C., Gosseries, O., ... Laureys, S. (2012). Functional neuroanatomy underlying the clinical subcategorization of minimally conscious state patients. Journal of Neurology, 259, 1087-1098.

Bruno, M.-A., Vanhaudenhuyse, A., Thibaut, A., Moonen, G., \& Laureys, S. (2011). From unresponsive wakefulness to minimally conscious PLUS and functional locked-in syndromes: recent advances in our understanding of disorders of consciousness. Journal of Neurology, 258(7), 1373-1384. http://doi.org/10.1007/s00415-011-6114-x

Chatelle, C., Bodien, Y. G., Carlowicz, C., Wannez, S., Charland-Verville, V., Gosseries, O., ... Giacino, J. T. (2016). Detection and Interpretation of Impossible and Improbable Coma Recovery Scale-Revised Scores. Archives of Physical 
Medicine and Rehabilitation, 97, 1295-1300.

http://doi.org/10.1016/j.apmr.2016.02.009

Cruse, D., Chennu, S., Chatelle, C., Bekinschtein, T. A., Fernández-Espejo, D., Pickard, J. D., ... Owen, A. M. (2011). Bedside detection of awareness in the vegetative state : a cohort study. The Lancet, 6736. http://doi.org/10.1016/S01406736(11)61224-5

Demertzi, A., Ledoux, D., Bruno, M.-A., Vanhaudenhuyse, A., Gosseries, O., Soddu, A., ... Laureys, S. (2011). Attitudes towards end-of-life issues in disorders of consciousness: a European survey. Journal of Neurology, 258, 1058-1065.

Demertzi, A., Schnakers, C., Ledoux, D., Chatelle, C., Bruno, M.-A., Vanhaudenhuyse, A., ... Laureys, S. (2009). Different beliefs about pain perception in the vegetative and minimally conscious states: A European survey of medical and paramedical professionals. In Progress in Brain Research (Vol. 177, pp. 329-338). Elsevier.

Dolce, G., Lucca, L. F., Candelieri, A., Rogano, S., Pignolo, L., \& Sannita, W. G. (2011). Visual pursuit in the severe disorder of consciousness. Journal of Neurotrauma, 28, 1149-1154.

Estraneo, A., Moretta, P., Cardinale, V., De Tanti, A., Gatta, G., Giacino, J. T., \& Trojano, L. (2014). A multicentre study of intentional behavioural responses measured using the Coma Recovery Scale-Revised in patients with minimally conscious state. Clinical Rehabilitation, 29(8), 803-808. http://doi.org/10.1177/0269215514556002

Giacino, J. T., Ashwal, S., Childs, N., Cranford, R., Jennett, B., Katz, D. I., ... Zasler, N. D. (2002). The minimally conscious state. Neurology, 58, 349-353.

Giacino, J. T., Kalmar, K., \& Whyte, J. (2004). The JFK Coma Recovery ScaleRevised: Measurement Characteristics and Diagnostic Utility. Archives of Physical Medicine and Rehabilitation, 85, 2020-2029.

Gill-Thwaites, H. (1997). The Sensory Modality Assessment Rehabilitation Technique A tool for assessment and treatment of patients with severe brain injury in a vegetative state. Brain Injury, 11(10), 723-34. http://doi.org/10.1080/026990597123098

Laureys, S., Celesia, G., Cohadon, F., Lavrijsen, J., Leon-Carrion, J., Sannita, W., ... 
Consciousness, the E. T. F. on D. of C. (2010). Unresponsive wakefulness syndrome: a new name for the vegetative state or apallic syndrome. $B M C$ Medicine, 8, 68 .

Lovstad, M., Froslie, K. F., Giacino, J. T., Skandsen, T., Anke, A., \& Schanke, A. K. (2010). Reliability and diagnostic characteristics of the JFK coma recovery scalerevised: exploring the influence of rater's level of experience. J Head Trauma Rehabil, 25(5), 349-356. http://doi.org/10.1097/HTR.0b013e3181cec841

Majerus, S., Bruno, M.-A., Schnakers, C., Giacino, J. T., \& Laureys, S. (2009). The problem of aphasia in the assessment of consciousness in brain-damaged patients. In Progress in Brain Research (Vol. 177, pp. 49-61). http://doi.org/10.1016/S0079-6123(09)17705-1

Noé, E., Olaya, J., Navarro, M. D., Noguera, P., Colomer, C., García-Panach, J., ... Ferri, J. (2012). Behavioral recovery in disorders of consciousness: A prospective study with the Spanish version of the coma recovery scale-revised. Archives of Physical Medicine and Rehabilitation, 93(3), 428-433.e12. http://doi.org/10.1016/j.apmr.2011.08.048

Sattin, D., Minati, L., Rossi, D., Covelli, V., Giovannetti, A. M., Rosazza, C., ... Leonardi, M. (2015). The Coma Recovery Scale Modified Score: a new scoring system for the assessment of patients with disorders of consciousness. International Journal of Rehabilitation Research, 38, 350-356. http://doi.org/10.1097/MRR.0000000000000135

Schnakers, C., Bessou, H., Rubi-Fessen, I., Hartmann, A., Fink, G. R., Meister, I., ... Majerus, S. (2015). Impact of Aphasia on Consciousness Assessment: A CrossSectional Study. Neurorehabilitation and Neural Repair, 29(1), 41-47. http://doi.org/10.1177/1545968314528067

Schnakers, C., Vanhaudenhuyse, A., Giacino, J., Ventura, M., Boly, M., Majerus, S., ... Laureys, S. (2009). Diagnostic accuracy of the vegetative and minimally conscious state: Clinical consensus versus standardized neurobehavioral assessment. BMC Neurology, 9(1), 35 .

Seel, R. T., Sherer, M., Whyte, J., Katz, D. I., Giacino, J. T., Rosenbaum, A. M., ... Zasler, N. (2010). Assessment Scales for Disorders of Consciousness: EvidenceBased Recommendations for Clinical Practice and Research. Archives of Physical 
Medicine and Rehabilitation, 91(12), 1795-1813.

Shiel, a, Horn, S. a, Wilson, B. a, Watson, M. J., Campbell, M. J., \& McLellan, D. L. (2000). The Wessex Head Injury Matrix (WHIM) main scale: a preliminary report on a scale to assess and monitor patient recovery after severe head injury. Clinical Rehabilitation, 14, 408-416. http://doi.org/10.1191/0269215500cr326oa

Stender, J., Gosseries, O., Bruno, M.-A., Charland-Verville, V., Vanhaudenhuyse, A., Demertzi, A., ... Laureys, S. (2014). Diagnostic precision of PET imaging and functional MRI in disorders of consciousness: A clinical validation study. The Lancet, 384(9942), 514-522.

Teasdale, G., \& Jennett, B. (1974). Assessment of coma and impaired consciousness. A practical scale. The Lancet, 2(7872), 81-84.

Thibaut, A., Chatelle, C., Wannez, S., Deltombe, T., Stender, J., Schnakers, C., ... Gosseries, O. (2015). Spasticity in disorders of consciousness: a behavioral study. European Journal of Physical \& Rehabilitation Medicine, 51(4), 389-397 9p. Retrieved from http://search.ebscohost.com/login.aspx?direct=true \&db=c8h\&AN=109576194\&la ng=es\&site=ehost-live

Thibaut, A., Chatelle, C., Ziegler, E., Bruno, M. A., Laureys, S., \& Gosseries, O. (2013). Spasticity after stroke: physiology, assessment and treatment. Brain Injury, 27(10), 1093-105. http://doi.org/10.3109/02699052.2013.804202

Thibaut, A., Deltombe, T., Wannez, S., Gosseries, O., Ziegler, E., Dieni, C., ... Laureys, S. (2015). Impact of soft splints on upper limb spasticity in chronic patients with disorders of consciousness: A randomized, single-blind, controlled trial. Brain Injury, 29(7-8), 830-6. http://doi.org/10.3109/02699052.2015.1005132

Wijdicks, E. F. M., Bamlet, W. R., Maramattom, B. V, Manno, E. M., \& McClelland, R. L. (2005). Validation of a new coma scale: The FOUR score. Annals of Neurology, 58(4), 585-93. http://doi.org/10.1002/ana.20611 
Table 1. Percentages of patients correctly diagnosed in a minimally conscious state if the Coma Recovery Scale-Revised assessment is limited to 3, 4 or 5 items that are the most frequently observed.

\begin{tabular}{|c|l|c|c|}
\hline $\begin{array}{c}\text { Number } \\
\text { of items }\end{array}$ & \multicolumn{1}{|c|}{ Included CRS-R items } & Percentage & Confidence interval \\
\hline 3 & $\begin{array}{l}\text { Movement to command, visual pursuit, } \\
\text { visual fixation }\end{array}$ & $91.13 \%$ & $87.80-94.47 \%$ \\
\hline 4 & $\begin{array}{l}\text { Movement to command, visual pursuit, } \\
\text { visual fixation, localization to noxious } \\
\text { stimulation }\end{array}$ & $97.16 \%$ & $95.21-99.11 \%$ \\
\hline 5 & $\begin{array}{l}\text { Movement to command, visual pursuit, } \\
\text { visual fixation, localization to noxious } \\
\text { stimulation, automatic motor reactions }\end{array}$ & $98.94 \%$ & $97.73-100 \%$ \\
\hline
\end{tabular}

Figure 1. Frequency of observation of each Coma Recovery Scale-Revised item indicating a minimally conscious state $(n=282)$.

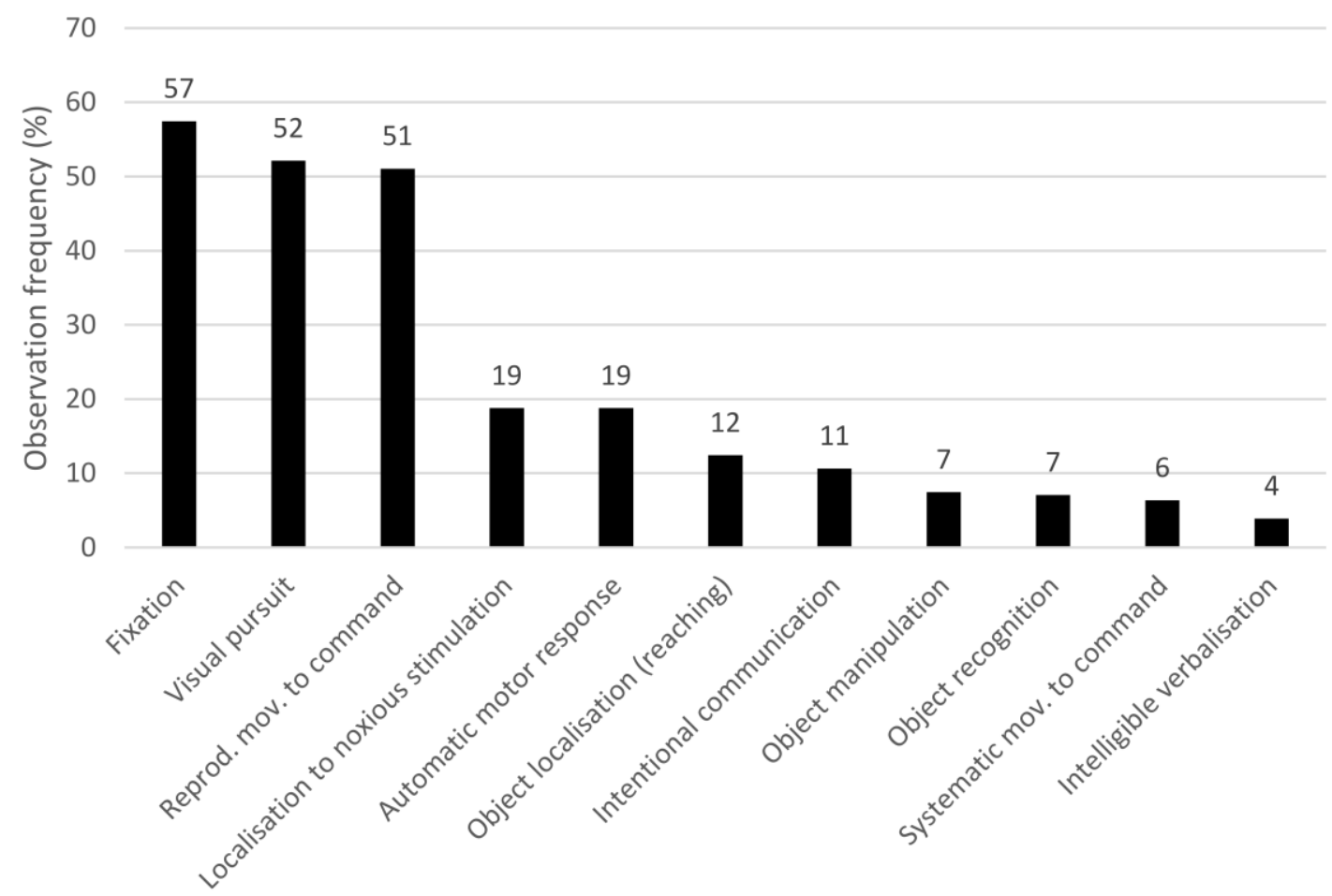

CRS-R items 
Figure 2. Frequency of observation of each Coma Recovery Scale-Revised item indicating a minimally conscious state, when only one sign of consciousness is shown by the patient during the behavioural assessment $(n=115)$.

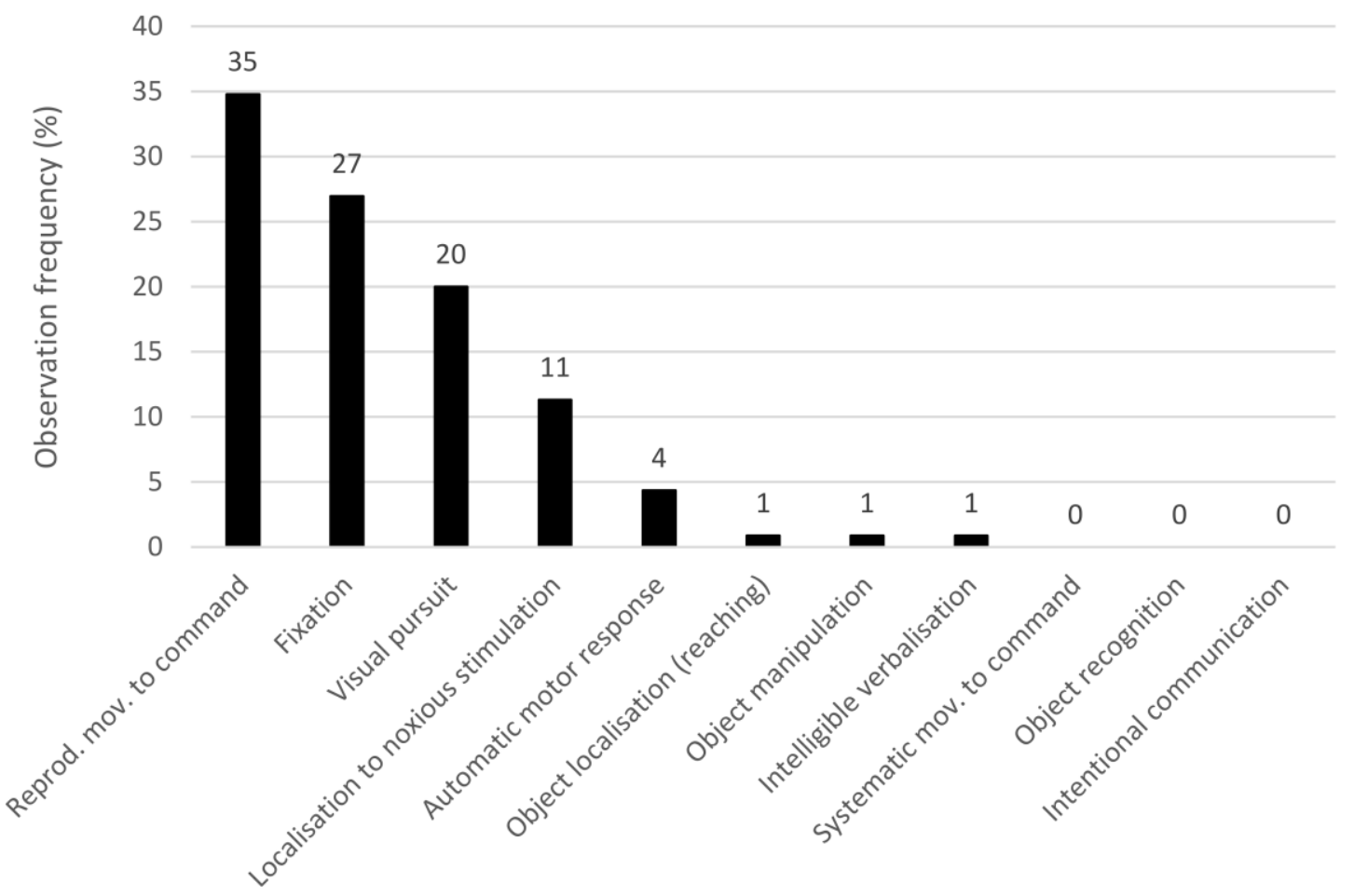

CRS-R items 